\title{
Mitochondrial Causes of Epilepsy: Evaluation, Diagnosis, and Treatment
}

\author{
Hannah E. Steele, MBBS, MRCP ${ }^{1,2}$ Patrick F. Chinnery, PhD, FRCP, FRCPath ${ }^{1,2}$ \\ ${ }^{1}$ Institute of Genetic Medicine, Newcastle University, Newcastle upon \\ Tyne, United Kingdom \\ 2 Department of Neurology, Royal Victoria Infirmary, Newcastle upon \\ Tyne, United Kingdom \\ Address for correspondence Patrick F. Chinnery, PhD, FRCP, FRCPath, \\ Institute of Genetic Medicine, International Centre for Life, Newcastle upon \\ Tyne, NE1 3BZ, United Kingdom (e-mail: patrick.chinnery@ncl.ac.uk). \\ Semin Neurol 2015;35:300-309.
Abstract Keywords
- epilepsy
- mitochondrial disease
- investigation
- diagnosis
- treatment \\ Mitochondrial disorders are frequently associated with seizures. In this review, the \\ authors discuss the seizure patterns and distinguishing features of mitochondrial \\ epilepsy, alongside the indications for investigating, and how to investigate epilepsy \\ from a mitochondrial perspective. Finally, they discuss management strategies for this \\ complex group of patients.
}

Seizures are a common and highly heterogeneous feature of mitochondrial disease. They can arise at any age, may be the presenting feature of the underlying biochemical defect, and can occur in the absence of a clear family history despite the genetic etiology. Furthermore, they are frequently a secondary feature in a complex phenotype; thus, detailed semiology is often lacking, ${ }^{1}$ although increasingly this is being addressed. ${ }^{2-7}$ The heterogeneous patient populations used in many of the series describing seizures add a further challenge to seizure interpretation. ${ }^{2,3,8-12}$

Ictal activity in mitochondrial disease may result from metabolic disturbance, encephalopathy, or an acquired structural lesion such as a stroke-like episode. However, seizures may occur without these factors. Furthermore, the mechanisms are not mutually exclusive. Accordingly, epilepsy in mitochondrial disease straddles the genetic and structural/ metabolic categories in the 2010 International League Against Epilepsy (ILAE) classification of the epilepsies outlined in -Table $\mathbf{1}$. $^{13}$

A large driver for the restructuring of this classification is the ongoing advance in genetic medicine and diagnostic technologies, ${ }^{13}$ exemplified by increasing descriptions of epilepsy causing gene variations ${ }^{14}$ and mirrored in the field of mitochondrial disease. ${ }^{15}$ As a combined consequence of the phenotypic diversity, the increasing genetic complexity and a historical paucity of detailed seizure semiology, the clinician is faced with a considerable challenge in identifying, classifying, and treating epilepsy arising due to mitochondrial disease.

The aim of this review is therefore to provide the nonspecialist reader with:

- An introduction to the etiology and clinical features of mitochondrial disorders

- An overview of epilepsy in the context of both syndromic and nonsyndromic mitochondrial disease

- A practical approach of when to consider a mitochondrial disorder in a patient with epilepsy

- A schema to investigate epilepsy with a suspected mitochondrial basis

- An overview of mitochondrial seizure management

\section{Mitochondrial Disease Overview}

Mitochondrial disorders are genetically determined metabolic diseases arising due to biochemical deficiency of the respiratory chain. They affect around 1 in 5,000 of the population in the United Kingdom (UK). ${ }^{16}$

The mitochondrial respiratory chain sits in the inner mitochondrial membrane and is responsible for the efficient generation of ATP through the process of oxidative phosphorylation (OXPHOS). The chain comprises five complexes, each with multiple subunits, which are coded for by both
Issue Theme Etiology of Epilepsy; Guest Editors: Philip Smith, MD, FRCP,

FAcadMEd, and Rhys Thomas, BSc, MRCP, MSc, PhD
Copyright @ 2015 by Thieme Medical Publishers, Inc., 333 Seventh Avenue,

New York, NY 10001, USA. Tel: +1(212) 584-4662. 
Table 1 International League Against Epilepsy classification

\begin{tabular}{|l|l|}
\hline Category & Discussion \\
\hline Genetic & $\begin{array}{l}\text { The epilepsy is a direct result of a known or presumed genetic defect(s) in which seizures are the core } \\
\text { symptom of the disorder. }\end{array}$ \\
\hline Structural/metabolic & $\begin{array}{l}\text { There is a distinct structural or metabolic condition or disease that has a substantially increased risk of } \\
\text { developing epilepsy. Structural lesions may be acquired or of genetic origin, e.g., tuberous sclerosis. In } \\
\text { contrast to genetic epilepsies, here there is a separate disorder interposed between the genetic defect } \\
\text { and the epilepsy. }\end{array}$ \\
\hline Unknown & The nature of the underlying cause is currently unknown. \\
\hline
\end{tabular}

mitochondrial and nuclear genomes. A relevant mutation in either genome may therefore compromise respiratory chain function with resultant cellular ATP deficiency. Consequently, the clinical features of mitochondrial disorders are most evident in tissues with high-energy demands, with the central and peripheral nervous systems being particularly susceptible.

Recognition of mitochondrial phenotypes may be complex. However, there are certain features (-Fig. 1) that suggest a bioenergetic deficit. Specific symptom combinations may enable a clinician to diagnose one of the canonical mitochondrial disorders such as MELAS (mitochondrial encephalomyopathy, lactic acidosis, and stroke-like episodes), or MERRF (myoclonic epilepsy and ragged-red fibers). However, many individuals are oligo-symptomatic and consequently do not fulfil requirements for a syndromic diagnosis.

\section{Epilepsy in Syndromic Mitochondrial Disease}

Epilepsy is a major phenotypic feature of several syndromic mitochondrial disorders, including Leigh syndrome, AlpersHuttenlocher syndrome, MELAS, and MERRF.

\section{Leigh Syndrome}

Leigh syndrome (LS) is a progressive, neurodegenerative syndrome characterized by impaired mitochondrial function

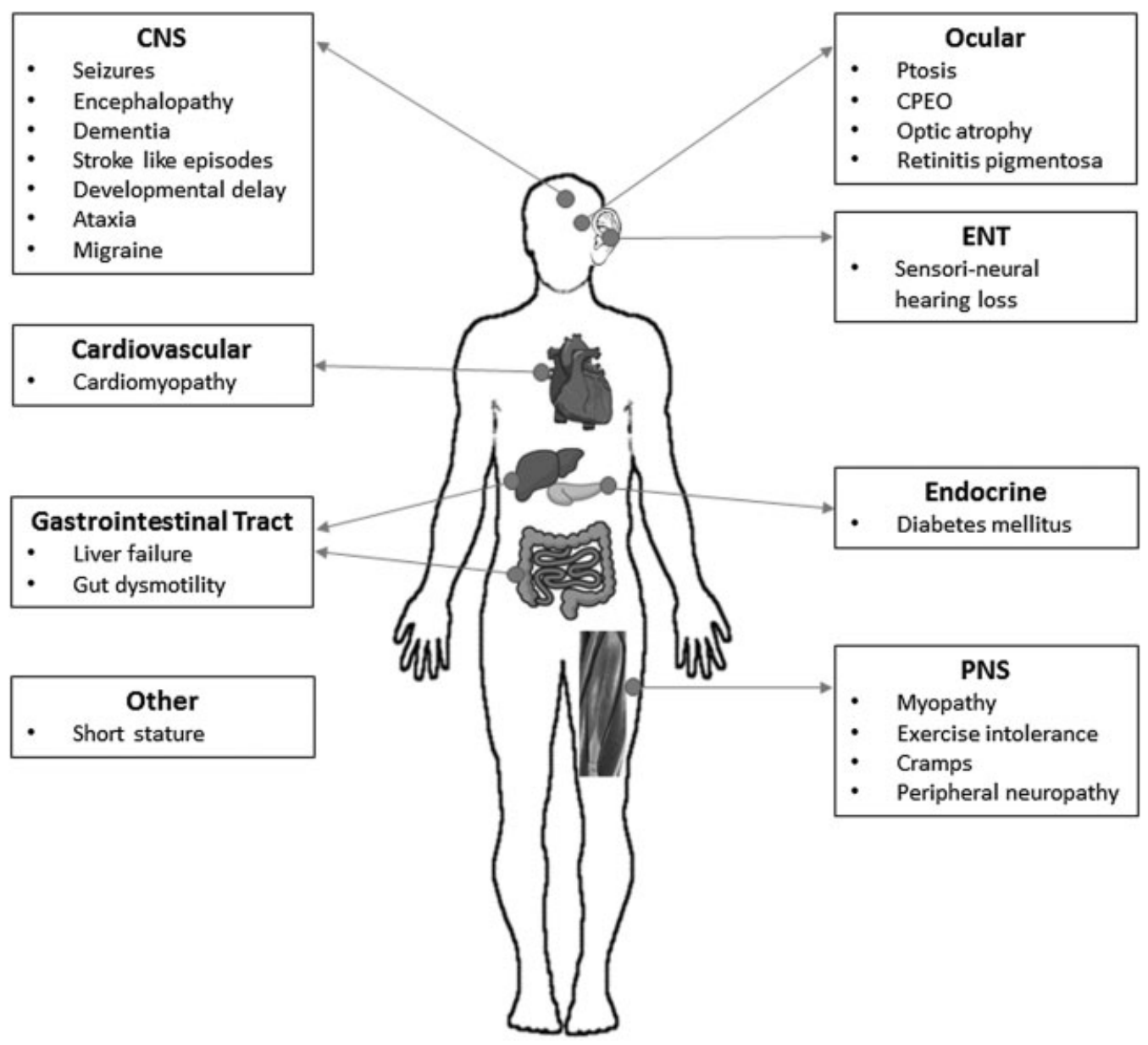

Fig. 1 An illustration of the common systemic features of mitochondrial disease. CNS, central nervous system; CPEO, chronic progressive ophthalmoplegia; ENT, ear, nose, and throat; PNS, peripheral nervous system. 
(OMIM 256000). The genetic basis of LS is complex, but approximately $50 \%$ of cases arise due to mutations in SURF1, a complex IV assembly gene. ${ }^{9}$

A striking feature of LS is the characteristic bilateral, focal neuropathological change evident on magnetic resonance (MR) brain imaging. It is the most common manifestation of mitochondrial disease in children, and symptom onset usually occurs between 3 and 12 months. However, the diagnosis should be considered in older individuals, including adults, with suggestive clinical features. ${ }^{17,18}$ Prognosis is poor, with death often occurring in early infancy.

Triad of Leigh syndrome:

1. Progressive neurodegeneration

2. Mitochondrial impairment

3. Characteristic bilateral central nervous system (CNS) lesions

Seizures occur in at least $40 \%$ of those with $\mathrm{LS}^{7,19}$ though some smaller case series report a prevalence of up to twice that. ${ }^{20,21}$ In a large natural history study of 130 patients with LS, epilepsy was the fourth most common clinical feature, after movement disorders, ocular involvement, and feeding difficulties, both at presentation and throughout the disease course. ${ }^{7}$ Over half of individuals with epilepsy due to LS have a generalized seizure disorder, with both myoclonic and absence seizures reported. ${ }^{7}$ This supports the findings of smaller series where myoclonic seizures are a frequent occurrence. $^{21,22}$ Focal seizures and infantile spasms are also recognized, but occur less often and may co-exist with generalized seizures. $^{7}$

Treatment of seizures associated with LS is difficult: They are frequently refractory (31-75\%) and a cause of death. ${ }^{7,23}$ Genetic heterogeneity may contribute to seizure risk, and there is emerging evidence that the presence of seizures in LS indicates a poor prognosis. ${ }^{7,23}$

Key Points: Leigh syndrome

- Triad of neurodegeneration, mitochondrial dysfunction, and characteristic symmetrical CNS imaging change

- Generalized seizures $>$ focal seizures

- Mainly presents in children, but consider in adults with appropriate clinical features

\section{Alpers-Huttenlocher Syndrome}

Alpers-Huttenlocher syndrome (AHS) is a life-limiting condition arising due to recessive mutations in the nuclear gene encoding the mitochondrial polymerase gamma (OMIM 203700). It causes a classical triad of intractable seizures with intellectual decline and liver dysfunction. It has a bimodal distribution of onset, with a first peak occurring in the preschool years, and a second in the late teens/early twenties. ${ }^{24}$

Seizures, including status epilepticus, are the primary feature of AHS in about half of patients, but for others, nonspecific features such as failure to thrive, may predominate initially. ${ }^{24}$ Seizure emergence heralds inexorable cognitive decline regardless of when in the disease course it occurs.
The onset is often explosive, and in common with LS, multiple seizure types often co-exist. However, in contrast to LS, focal seizures and myoclonus are most frequent in AHS. ${ }^{25-27}$ Progression to epilepsia partialis continua (EPC) and secondary generalized status epilepticus is characteristic and refractory seizures are a recognized cause of death..$^{24,25,28,29}$ There are no disease-modifying treatments available. Consequently, clinicians should anticipate the need for palliative care provision. The use of sodium valproate is contraindicated due to the occurrence of fatal hepatotoxicity.

\section{Key points: Alpers-Huttenlocher syndrome}

- Triad of intractable seizures, intellectual decline, and liver dysfunction

- Focal seizures and myoclonus $>$ generalized seizures

- Avoid sodium valproate use

\section{MELAS Due to $\mathrm{mt} .3243 \mathrm{~A}>\mathrm{G}$ Mutation}

The mt.3243A $>G$ mutation in the mitochondrial DNA (mtDNA) MTTL1 gene is the most frequently identified mutation causing MELAS. Other phenotypes may also arise due to this point mutation, such as CPEO (chronic progressive external ophthalmoplegia) and MIDD (maternally inherited diabetes and deafness). Conversely, MELAS may also be caused by other mtDNA mutations, ${ }^{30}$ and has been described in association with nuclear DNA mutations such as POLG. ${ }^{31,32}$

In a cohort of individuals carrying the mt.3243A $>G$ mutation, $24 \%$ of all symptomatic individuals manifested seizures. ${ }^{1}$ There was a clear relationship between phenotype and seizure prevalence, with 50 to $62 \%$ of those with MELAS and MELAS overlap syndromes (MELAS with either MIDD or CPEO) experiencing seizures. In comparison, seizure prevalence was $25 \%$ in those with mixed neurologic features, $17 \%$ with MIDD/CPEO overlap, and just $8 \%$ with MIDD alone. ${ }^{1}$

For those with mt.3243A > G MELAS, focal seizures and prolonged focal status epilepticus encompassing occipital lobe status, EPC, hemiclonic status, nonconvulsive status, and spike-wave stupor dominate the clinical picture. ${ }^{4,33}$ However, truly generalized seizures are reported in a minority. ${ }^{4}$ Therefore, mt.3243A > G MELAS should be considered a disorder in which focal and primary generalized seizures can manifest, although there are few reports to date of this occurring in the same individual. ${ }^{2-4}$

Electroencephalogram (EEG) changes such as focal slowing or epileptiform changes are rather nonspecific, but tend to show a posterior predilection. ${ }^{4}$ In contrast, it is reported that periodic lateralized epileptiform discharges (PLEDS) in children have a limited differential diagnosis, with MELAS being a possibility. ${ }^{4}$

It has been proposed that seizures in mt.3243A $>G$ MELAS may occur in two distinct and predictable patterns. ${ }^{4}$ In some individuals, seizures appear solely in the context of stroke-like episodes, and in others seizures also occur at other times. The implication of this observation for seizure management remains unclear-in particular whether select individuals require antiepileptic drugs only at times of metabolic crises. However, as the numbers reported to date are small, this observation requires substantiation in larger cohorts. 
Key Points: mt.3243A > G MELAS

- $50 \%$ individuals with mt.3243A > G MELAS have epilepsy

- Focal seizures predominate and may be prolonged

- Primary generalized seizures may also manifest

\section{MERRF Due to mt.8344A > G Mutation}

Myoclonic epilepsy with ragged-red fibers is characterized by myoclonic epilepsy, generalized seizures, particularly clonic, and ataxia. $^{3}$ It most frequently arises due to the mtDNA mt.8344A > G point mutation in MTTK, although there are also other pathogenic mutations. ${ }^{34,35}$ Myoclonic epilepsy with ragged-red fibers is one of the progressive myoclonic epilepsies that are associated with progressive intellectual decline and refractory seizures.

In contrast to the seizure phenomenology in mt.3243A > G MELAS, generalized seizures predominate in MERRF. Indeed, in a large genotype-phenotype analysis of the mt.8344A > G mutation, focal seizures were not reported. ${ }^{6}$ However, 25 to $35 \%$ of patients with mt.8344 MERRF experienced generalized seizures (not otherwise specified), and 35 to $45 \%$ experienced myoclonus. Furthermore, they are frequently the reason an individual comes to medical attention, again in contrast with mt.3243 MELAS, where other clinical features such as stroke-like episodes are more commonly the reason for medical attention. Around one in three people with MERRF present before the age of 16 years. ${ }^{6}$ Additional systemic features co-exist in many patients with weakness (59\%), hearing loss (35\%), ataxia (23\%), raised serum creatine kinase (44\%), and lipomatosis (32\%) being notably common. ${ }^{6}$

The interictal EEG may be normal, but often shows slow background activity in conjunction with generalized epileptiform discharges, such as spikes, polyspike, and irregular and slow wave complexes. ${ }^{5}$ Hyperventilation is safe, ${ }^{36}$ and photic stimulation may enhance generalized discharges. ${ }^{3,5}$

Key Points: mt.8344A > G MERRF

- Generalized seizure disorder

- Valproate can be a first-line treatment

\section{Mixed Syndromic and Nonsyndromic Disorders}

\section{Recessive Polymerase Gamma- (POLG-) Related Disorders}

Polymerase gamma is a DNA polymerase responsible for mtDNA repair and replication. Although POLG is encoded by nuclear DNA, it is the only DNA polymerase found in mitochondria; hence, POLG disorders are characterized by multiple mtDNA deletions that accumulate over time. Consequently, highly variable clinical features may arise due to either dominant or recessive mutations. ${ }^{37}$

Although CPEO characterizes the dominant disorders, the recessive disorders are more complex. There are several syndromes including mitochondrial recessive ataxia syndrome (MIRAS); myoclonic epilepsy myopathy sensory ataxia (MEMSA); spinocerebellar ataxia with epilepsy (SCAE); sensory ataxia with neuropathy, dysarthria, and ophthalmoplegia (SANDO); as well as AHS, described earlier. However, as many patients do not fulfill requirements for a syndromic diagnosis, particularly early in the disease course, ${ }^{38,39}$ much of the literature pertaining to recessive POLG seizure manifestations considers these syndromes together. Consequently, our discussion reflects this.

Seizures are a common manifestation of the recessive POLG mutations, occurring in approximately $40 \%$ of those presenting in adulthood, and up to $85 \%$ of those presenting under the age of 5 years. ${ }^{38}$ Although pathogenic mutations throughout the gene are reported, ${ }^{38,40}$ two particular mutations are common in the European population: $1399 \mathrm{G}>\mathrm{A}$ (p. A467T) and 2243G > C (p.W748S). ${ }^{41}$ Individuals with compound heterozygous or homozygous changes in these variants have a mean age of symptom onset of 18 years. In over half of these people (63\%), seizures are the presenting feature of the disorder. ${ }^{42}$

The hallmark of POLG-related seizures is progressive focal motor seizures, particularly affecting the upper limb, neck, or proximal trunk. Myoclonus is common, as are episodes of convulsive and nonconvulsive status epilepticus, including epilepsia partialis continua. Epilepsia partialis continua may remain focal for many years, but invariably progresses to refractory epileptic encephalopathy. Status epilepticus is almost ubiquitous. ${ }^{39,42}$ Seizure onset is frequently explosive and progression fulminant. ${ }^{39}$

One clinical feature suggestive of $P O L G$-related seizures is the occipital predilection in the early stages, characterized by visual disturbances including colored lights, scotoma, or visual blurring. ${ }^{39,42}$ Many patients develop additional neurologic features as their condition progresses. ${ }^{39}$ These include migraine, neuropathy, ataxia, and chronic progressive external ophthalmoplegia, clear indicators of a complicated epilepsy and ultimately of the underlying molecular diagnosis.

In addition to the clinical features, there are specific features in the EEG that suggest POLG mutations. These include rhythmic high amplitude delta with superimposed spikes and polyspikes (RHADS) ${ }^{27}$ and asymmetric occipital lobe or central polyspikes/polysharp complexes with associated slowing. ${ }^{26}$ Electroencephalogram changes correlate with magnetic resonance imaging (MRI) changes as well as the clinical epileptiform features. ${ }^{39,42}$ The posterior predominance of seizure semiology, as seen in the EEG and MRI, reflects high metabolic activity in the occipital lobes rendering them vulnerable to the effects of bioenergetic dysfunction. ${ }^{43}$

Disease mortality in recessive POLG disorders is correlated with seizure severity and progression, and as with the other disorders mentioned above, status epilepticus is a common end-of-life event. ${ }^{39}$ There is evidence that the genotype has an impact on survival, as those with compound heterozygous changes have a poorer prognosis than those with homozygous changes at either of the common European mutation sites described above. ${ }^{39,40}$

\section{Key Points: Recessive POLG mutations}

- Seizures affect $40 \%$ of people presenting in adulthood and up to $85 \%$ of those presenting in childhood

- Occipital predilection early in disease course 
- Prolonged focal motor seizures characteristic

- Avoid sodium valproate due to risk of liver failure

\section{Twinkle (C10orf2) Mutations}

Twinkle (C10orf2) is a nuclear-encoded mitochondrial replicase. Mutations in twinkle result in the accumulation of mitochondrial DNA depletion, and consequently, the clinical features associated with these mutations mimic those seen with POLG. Chronic progressive external ophthalmoplegia arises due to dominant mutations in twinkle, whereas infantile-onset spinocerebellar ataxia (IOSCA) occurs with recessive mutations.

Seizures are seen in most of those with IOSCA (18/21 patients). ${ }^{44}$ Typically, focal seizures and myoclonic jerks occur initially and then progress to epilepsia partialis continua and generalized status. Seizures are often triggered by infection or surgery, and are a common cause of death. Again, in common with POLG seizure disorders, PLEDs were identified on the EEG and progression of EEG findings was noted with disease progression. $^{44}$

\section{Epilepsy in Nonsyndromic Mitochondrial Disease}

Nonsyndromic mitochondrial disease occurs frequently-particularly early in the disease course. Reflecting this, $50 \%$ to $70 \%$ of children manifesting seizures in the context of a respiratory chain defect (RCD) have a nonsyndromic diagnosis. ${ }^{2,3,11,12}$ In contrast, similar adult patients have nonsyndromic features in approximately $40 \%$ of cases. ${ }^{3}$

Chevellier studied 165 adults and children with confirmed mitochondrial disease, of whom roughly a third $(n=60)$ had had a previously identified seizure. ${ }^{2}$ Most patients included in the study had neither a (mitochondrial) syndromic (68\%) nor molecular genetic diagnosis (72\%). Perhaps surprisingly, $70 \%$ of this population was well controlled with treatmentalthough the proportion of these with and without a mitochondrial syndromic diagnosis is unknown. This is in keeping with the proportion of individuals with epilepsy of all causes who can achieve seizure freedom with appropriate medication. $^{45}$

\section{Mitochondrial Syndromes Associated with Infrequent Seizures}

In contrast to the conditions described above, seizures are an uncommon phenotypic feature of many other classical mitochondrial disorders, including

- Neuropathy, ataxia, retinitis pigmentosa (NARP) ${ }^{46}$

- Kearns-Sayre syndrome (KSS) ${ }^{47,48}$

- Leber's hereditary optic neuropathy (LHON) 49,50

- Maternally inherited diabetes and deafness (MIDD) ${ }^{1}$

\section{When to Consider a Mitochondrial Disorder in a Patient with Epilepsy}

It seems highly likely that epilepsy arising due to mitochondrial disease is underrecognized, given the estimated preva- lence of mitochondrial disorders, ${ }^{16}$ the oligosymptomatic basis of many patients with mitochondrial disease, ${ }^{1,51}$ the often long diagnostic odyssey, and the impact of active investigation in other mitochondrial disorders. ${ }^{51}$ Here, we flag clinical features that may indicate an underlying mitochondrial etiology in a patient with a seizure disorder. However, these are by no means diagnostic and alternative pathologies should also be contemplated in the diagnostic workup.

\section{Clinical Features}

Epilepsy in Association with Undiagnosed Multisystem Disease

Individuals with epilepsy and two or more of the clinical features below, without other unifying diagnosis, should be tested for the mt.3243A > G mutation in the first instance, ${ }^{1}$ before moving on to consider other appropriate investigations, outlined later.

- Cardiomyopathy

- Deafness

- Developmental delay or cognitive decline

- Diabetes mellitus

- Gastrointestinal disturbance (constipation and/or irritable bowel syndrome)

- Migraine

- Chronic progressive external ophthalmoplegia

- Retinopathy

There should be a low threshold for POLG testing for people presenting with seizures and liver dysfunction.

\section{Complex Childhood-Onset Epilepsy}

Individuals with childhood-onset epilepsy ultimately diagnosed with a respiratory chain defect often experience preceding symptoms of failure to thrive, psychomotor delay, ataxia $^{8}$; encephalopathy, multiorgan symptomatology, or a fluctuating clinical course. ${ }^{10}$ Furthermore, mitochondrial disorders are a recognized cause of epileptic encephalopathy ${ }^{44,52}$ : conditions where the seizures themselves are thought to contribute to intellectual decline.

\section{Family History}

Our clinical experience mirrors reports of patients describing seemingly disparate clinical presentations in family members, which taken together, clinically suggest a unifying mitochondrial disorder. $4,5,7,10$

\section{Features of the Epilepsy Itself}

\section{Status Epilepticus}

Mitochondrial diseases are a key consideration in recurrent or refractory episodes of convulsive or nonconvulsive status epilepticus in adults or children. $4,7,8,23-25,28,33,39,42$

\section{Epilepsia Partialis Continua}

Epilepsia partialis continua is characterized by persistent focal seizures with retained consciousness. It is a feature of 
several mitochondrial disorders, including mt.3243A $>\mathrm{G}$ MELAS $^{4}$ (often in association with stroke like episodes) and AHS. $^{26}$ It can also occur with twinkle $\left(\right.$ C10orf12) ${ }^{44}$ and POLG ${ }^{39,42}$ mutations. Although it has been described on occasion in $\mathrm{LS}^{53}$ this does not appear to be a common feature of the disorder. $7,19,20$

\section{Occipital Lobe Epilepsy}

Occipital lobe epilepsy is an uncommon clinical presentation, affecting $2 \%$ to $13 \%$ of those with focal epilepsies. ${ }^{54}$ Occipital lobe epilepsy can arise due to recognized electroclinical syndromes, as well as symptomatic causes ${ }^{55}$; however, seizures associated with mt.3243A > G MELAS and POLG have a clearly documented posterior predilection-particularly early in the disease course. ${ }^{4,33,42}$

\section{Myoclonic Epilepsy}

Myoclonic epilepsy is a cardinal feature of MERRF, ${ }^{5,6}$ and widely recognized in other mitochondrial disorders. ${ }^{10,56}$ Typically, mitochondrial myoclonic seizures are associated with clinical progression or other parameters of complex disease, setting them apart from the benign childhood and juvenile myoclonic epilepsies. Other causes of progressive myoclonic epilepsy should be considered. ${ }^{57}$

\section{Recognized Electroclinical Syndrome}

The following electroclinical syndromes have been reported in several cohorts to have a mitochondrial contribution:

- Ohtahara syndrome 12,58

- West syndrome - $^{12,59}$

- Landau-Kleffner syndrome $e^{10,12,60}$

- Lennox-Gastaut syndrome $e^{10,12}$

Other electroclinical syndromes associated with mitochondrial diseases occur less frequently and are reviewed elsewhere. ${ }^{9}$

\section{Investigative Features}

\section{Electroencephalogram}

There is not a single "characteristic mitochondrial EEG." Indeed, the most frequently identified abnormalities are nonspecific and include generalized slowing (60\%), ${ }^{2,11}$ multifocal discharges, focal discharges or generalized discharges (all 40\%). ${ }^{2}$ Photosensitivity has been reported in patients with both MELAS and MERRF, 3,5 and although hyperventilation is safe in this patient cohort, it does not appear to increase the diagnostic yield. ${ }^{36}$ Nonetheless, despite these nonspecific features, the EEG may show traits suggesting a mitochondrial disorder. These include:

- EEG abnormalities with a posterior predilection ${ }^{4,39,42}$

- Periodic lateralized epileptiform discharges (PLEDS) in children are suggestive of MELAS ${ }^{4}$

- Focal high-voltage delta waves with polyspikes (FHDPS) may occur in acute stroke-like lesions in MELAS-although the other clinical features may prompt the diagnosis, rather than the $\mathrm{EEG}^{61}$
- Rhythmic high amplitude delta with superimposed spikes and polyspikes (RHADS) strongly suggest POLG mutations causing AHS. ${ }^{26,27}$

Few definitive studies have examined EEG changes throughout the disease course, but progression is a feature. $^{39,61}$

\section{Neuroimaging}

Brain imaging may be normal, particularly in nonsyndromic mitochondrial disease ${ }^{11}$ or early in the disease course. ${ }^{42}$ In one series, over half of patients with MERRF had normal cerebral imaging. ${ }^{6}$ However, several features have been identified frequently in those with respiratory chain defects and seizures, including prominent and progressive cerebral atrophy, ${ }^{5,6,8,10,11,42}$ cortical signal change, ${ }^{5,6}$ stroke-like episodes, ${ }^{4-6,33}$ and symmetrical extracortical lesions (such as signal change within the brainstem, basal ganglia, or thalamus). ${ }^{11,39,42}$

\section{How Should I Investigate?}

The ultimate aim of investigation is to reach a molecular genetic diagnosis. This obviates the need for ongoing investigation, aids management and follow-up, and enables individuals and their families to access appropriate genetic counselling. Although genetic testing of symptomatic individuals can be undertaken by general pediatricians or neurologists, the detection and interpretation of mtDNA mutations is particularly challenging due to the effects of heteroplasmy. Heteroplasmy describes a cardinal feature of mitochondrial disorders, whereby mtDNA mutation loads are carried at different levels in different tissues. This contributes to the highly variable phenotypic features of mitochondrial disorders, even within families. Consequently, if the diagnostic tissue has a low mutant mtDNA heteroplasmy level, the mutation may not be identified. Furthermore, the identification of a mtDNA mutation has particularly complex implications for relatives, and genetic counseling for family members is usually best undertaken by those with specific expertise. ${ }^{62}$

\section{Syndromic Presentations}

The approach to the investigation of a person with a syndromic presentation is relatively straightforward: appropriate targeted genetic testing.

\section{Nonsyndromic Presentations}

Many patients have nonsyndromic presentations, however, and for them the diagnostic process is often longer (- Fig. 2). Many will undergo muscle or skin biopsy looking for biochemical evidence of mitochondrial dysfunction, and to help target genetic testing. Although invasive, it is well tolerated, has a low complication rate, and can be done under local anesthesia as a day case procedure (in adult patients). Samples provide invaluable information regarding the structure (histochemistry) and function (respiratory chain enzyme analysis) of muscle. Furthermore, muscle tissue can be used 


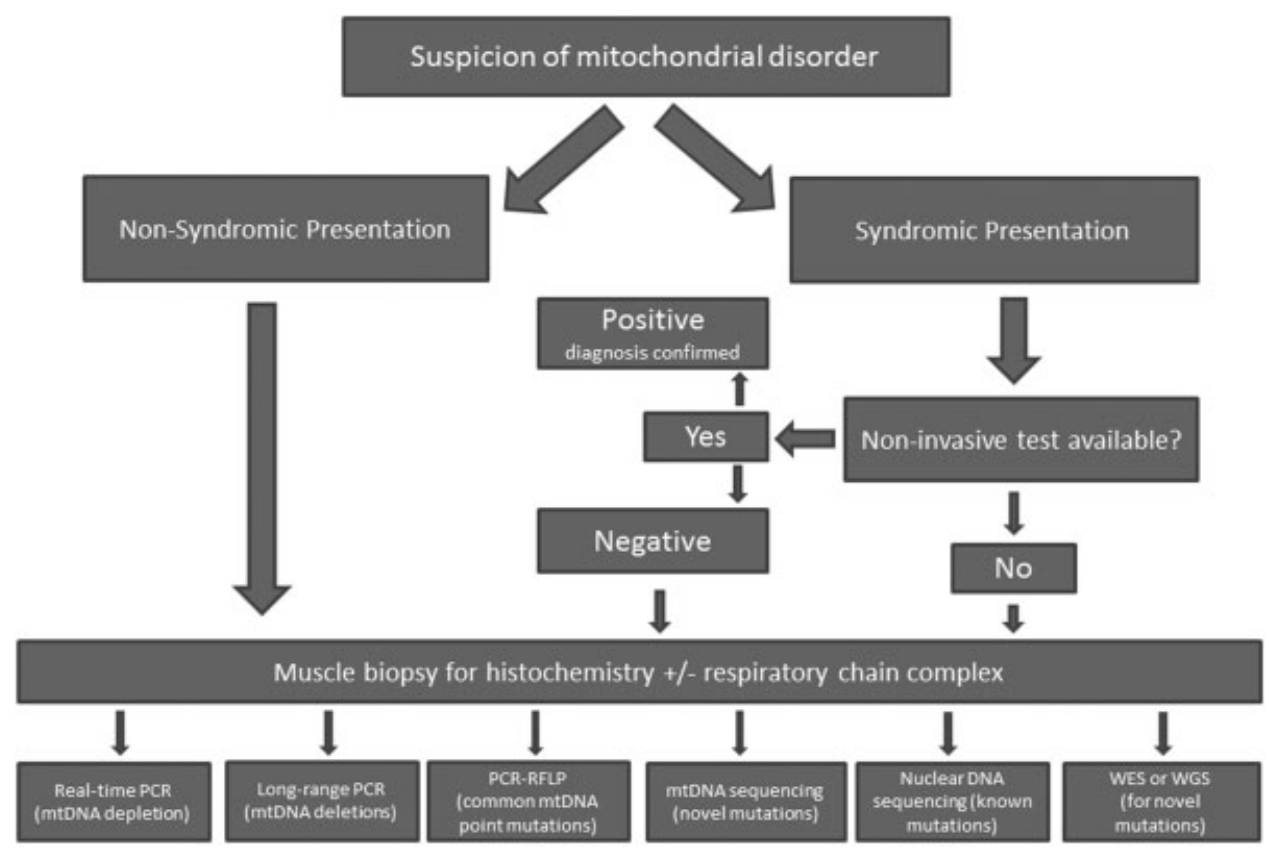

Fig. 2 A schematic of the investigative approach to suspected mitochondrial disorders. mtDNA, mitochondrial DNA; PCR, polymerase chain reaction; RFLP, restriction fragment length polymorphism; WES, whole exome sequencing; WGS, whole genome sequencing.

for further genetic analysis, as determined by the clinical features.

\section{Future Direction}

The impact of next-generation sequencing techniques on diagnosis will be determined as this becomes increasingly available at academic centers. ${ }^{15}$ In addition, diagnostic biomarkers, as well as markers of disease progression are an area of active research. This is particularly salient as disease-modifying treatments are developed, and established markers of respiratory chain dysfunction such as lactate and pyruvate are neither sensitive nor specific. ${ }^{63}$ Although fibroblast growth factor-21 (FGF-21) has shown some promise as a diagnostic tool, its use is not embedded in clinical practice. ${ }^{64,65}$

\section{Management of Mitochondrial Epilepsy}

\section{Supportive Care}

As seizures in mitochondrial disorders may be triggered, or exacerbated, by metabolic disturbance, physicians should aim to regulate the biochemical milieu by ensuring appropriate hydration, normalizing blood glucose, managing acidosis, and treating concomitant infections where present. ${ }^{66}$

Mitochondrial "cocktail" therapy consisting of co-enzyme $\mathrm{Q}_{10}$, vitamin $\mathrm{B}$ complex, vitamins $\mathrm{C}$ and $\mathrm{E}$, and L-carnitine has been used to treat a variety of respiratory chain defects. In one study, the carers of 48 children with seizures and a RCD were asked to report changes in seizure rate, behavior, and development. ${ }^{12}$ Although $75 \%$ reported improvements, the study was not primarily designed to assess drug efficacy. Furthermore, the 2012 Cochrane review did not identify any evidence-based disease modifying treatments for mitochondrial disease. $^{67}$
The remaining sections therefore focus on the symptomatic treatment of seizures arising in the context of respiratory chain disorders.

\section{Antiepileptic Drugs}

There are few high quality trials to inform best use of antiepileptic medications in mitochondrial disease specifically. However, in common with epilepsies arising from other causes, the choice of medication should largely depend upon whether the seizure disorder has a focal or generalized onset.

A wide range of antiepileptic drugs (AEDs) are used to control seizures in mitochondrial disease, including carbamazepine, clobazam, clonazepam, lamotrigine, levetiracetam, oxcarbazepine, phenobarbital, topiramate, and sodium valproate. Many patients are on more than a single drug., 811 Furthermore, treatment efficacy is difficult to assess due to the lack of trials and the heterogeneous nature of seizures in mitochondrial disease, which are often progressive.

In one series of adults and children, those with seizures were identified from a population with diagnosed respiratory chain disorders. Of these, $72 \%$ were "easily controlled" with AEDs and $28 \%$ were refractory. ${ }^{2}$ In contrast, other series report that 5 to $15 \%$ achieve seizure freedom with antiepileptic medication, ${ }^{8,11}$ highlighting the highly variable prognosis. Although this probably reflects different patient selection methods between series, it serves to emphasize that seizure freedom is a realistic and achievable goal for select patients with RCDs.

In addition to considering efficacy, clinicians should be aware of potential interactions between antiepileptic medications and mitochondrial function. Although many are described, most have no implications for widespread clinical practice. $^{68}$ The exception to this is use of sodium valproate 
use in those with a polymerase gamma (POLG) mutation, which may precipitate irreversible liver failure. Consequently, there is a move to screen for POLG mutations before starting it in children with refractory epilepsy. ${ }^{28}$ It seems appropriate to test adults in appropriate clinical circumstances.

\section{Intensive Care Support}

Status epilepticus or epilepsia partialis continua may require intensive care support for induction of anesthetic coma. There is no evidence base to guide specific treatment, and it is likely that a combination of antiepileptic medications will be used. Magnesium infusion is recommended as a treatment for super-refractory status epilepticus ${ }^{69}$ and its use in status epilepticus due to recessive POLG mutations has been described in two cases, both of whom showed improvement in seizure control. ${ }^{70}$

The prognosis following intensive care unit (ICU) admission for seizures has not been well reported in the context of mitochondrial disease specifically. In a series of 11 patients with mitochondrial disorders admitted to an ICU with seizures, 4 died, suggesting the prognosis is poor. ${ }^{71}$ This is largely in keeping with figures reported elsewhere for the outcome of individuals following status epilepticus. ${ }^{72}$ Therefore, endof-life care is a salient issue to consider for such patients, even while active management is ongoing.

\section{Ketogenic Diet}

The ketogenic diet has an increasing evidence base for use in mitochondrial disease and may have applications in the treatment of seizure disorders arising due to either nuclear or mitochondrial DNA defects. $8,12,73,74$ Predicting who will respond to ketogenic diet is not currently possible. However, in one study, $75 \%$ of individuals had a reduction in seizures of more than $50 \%$, and half of people became seizure free. ${ }^{12}$ Furthermore, ketogenic diet may be a useful adjunct to traditional pharmaceutical agents in the acute setting, ${ }^{73,75}$ although this effect is not universal and reporting bias is likely to be evident. ${ }^{42}$ Whether the ketogenic diet modifies the prognosis or disease course of mitochondrial disease is currently unknown. Specialist dietician input is required, and further support can be found from Matthew's Friends (in the UK)-a support group for those embarking on the ketogenic diet. ${ }^{76}$

\section{Surgical Management}

The current evidence base for the surgical management of mitochondrial seizures is limited to case reports and small case series. In the case of vagus nerve stimulation, five children with an electron transport chain deficiency did not experience seizure reduction following vagus nerve stimulator implantation. ${ }^{77}$ However, the patient population was small and heterogeneous, both in terms of the underlying genetic etiology and the seizure type. As such, it has limited ability to guide clinical application.

Traditional epilepsy surgery programs are usually inappropriate for those with mitochondrial disorders. However, palliative hemispherectomy in AHS has been described, ${ }^{29}$ which enabled a patient to leave intensive care and die at home. The report was well received $^{78,79}$ and as refractory seizures are a common end-stage feature of mitochondrial disease, select cases may benefit from such an approach.

\section{Palliative Care}

As mentioned, many mitochondrial disorders cause premature death, ${ }^{7,8,24}$ and for those with seizures, many deaths occur due to neurologic decompensation. ${ }^{8}$ Accordingly, palliative care requirements should be anticipated by the clinical team. ${ }^{80,81}$

\section{Summary}

Epilepsy is a common feature of mitochondrial disorders, occurring in approximately one in three of all people with a confirmed respiratory chain defect. ${ }^{2}$ Although seizures are highly heterogeneous in both manifestation and severity, there are often additional clinical indicators to suggest underlying mitochondrial dysfunction. Furthermore, although catastrophic (often) early-onset seizure disorders arise due to mitochondrial disease, for many individuals, seizures can be successfully managed with routine antiepileptic drugs. ${ }^{2}$

The investigation of suspected mitochondrial disease may be complex, particularly in nonsyndromic presentations. However, the increasing integration of next-generation sequencing technologies into clinical practice is likely to revolutionize the current diagnostic pathway. Far from removing clinicians from this diagnostic process, accurate interpretation of genetic variants requires skilled clinical phenotyping, and as such, physicians' clinical skills will continue to be needed at the "coal face."

Although approaches to prevent the transmission of mitochondrial disorders are being actively developed, these will not be appropriate in all circumstances. ${ }^{82}$ As such, the need for disease-modifying treatments in mitochondrial disorders will remain a priority for both those working in the field, and those affected by the disorders. Identifying the clinical features and genetic basis of mitochondrial disorders is just the first step in this process. Multicenter international collaboration will be required to conduct natural history studies of genetically defined cohorts, and thereby ensure the scientific robustness of future clinical trials. ${ }^{83}$

\section{Acknowledgments}

PFC is an Honorary Consultant Neurologist at Newcastle upon Tyne Foundation Hospitals NHS Trust, is a Wellcome Trust Senior Fellow in Clinical Science (101876/Z/13/Z), and a UK NIHR Senior Investigator. PFC receives additional support from the Wellcome Trust Centre for Mitochondrial Research (096919Z/11/Z), the Medical Research Council (UK) Centre for Translational Muscle Disease research (G0601943), and EU FP7 TIRCON, and the National Institute for Health Research (NIHR) Newcastle Biomedical Research Centre based at Newcastle upon Tyne Hospitals NHS Foundation Trust and Newcastle University. 


\section{References}

1 Nesbitt V, Pitceathly RD, Turnbull DM, et al. The UK MRC Mitochondrial Disease Patient Cohort Study: clinical phenotypes associated with the m.3243A $>\mathrm{G}$ mutation-implications for diagnosis and management. J Neurol Neurosurg Psychiatry 2013;84(8): 936-938

2 Chevallier JA, Von Allmen GK, Koenig MK. Seizure semiology and EEG findings in mitochondrial diseases. Epilepsia 2014;55(5): 707-712

3 Canafoglia L, Franceschetti S, Antozzi C, et al. Epileptic phenotypes associated with mitochondrial disorders. Neurology 2001;56(10): 1340-1346

4 Demarest ST, Whitehead MT, Turnacioglu S, Pearl PL, Gropman AL. Phenotypic analysis of epilepsy in the mitochondrial encephalomyopathy, lactic acidosis, and strokelike episodes-associated mitochondrial DNA A3243G mutation. J Child Neurol 2014;29(9): 1249-1256

5 Lorenzoni PJ, Scola RH, Kay CS, Arndt RC, Silvado CE, Werneck LC. MERRF: Clinical features, muscle biopsy and molecular genetics in Brazilian patients. Mitochondrion 2011;11(3):528-532

6 Mancuso M, Orsucci D, Angelini C, et al. Phenotypic heterogeneity of the 8344A>G mtDNA "MERRF" mutation. Neurology 2013; 80(22):2049-2054

7 Sofou K, De Coo IF, Isohanni P, et al. A multicenter study on Leigh syndrome: disease course and predictors of survival. Orphanet J Rare Dis 2014;9:52

8 El Sabbagh S, Lebre AS, Bahi-Buisson N, et al. Epileptic phenotypes in children with respiratory chain disorders. Epilepsia 2010;51(7): 1225-1235

9 Finsterer J, Zarrouk Mahjoub S. Mitochondrial epilepsy in pediatric and adult patients. Acta Neurol Scand 2013;128(3):141-152

10 Kang HC, Kwon JW, Lee YM, Kim HD, Lee HJ, Hahn SH. Nonspecific mitochondrial disease with epilepsy in children: diagnostic approaches and epileptic phenotypes. Childs Nerv Syst 2007;23(11): 1301-1307

11 Lee HF, Chi CS, Tsai CR, Chen CH. Epileptic seizures in infants and children with mitochondrial diseases. Pediatr Neurol 2011;45(3): 169-174

12 Lee YM, Kang HC, Lee JS, et al. Mitochondrial respiratory chain defects: underlying etiology in various epileptic conditions. Epilepsia 2008;49(4):685-690

13 Berg AT, Berkovic SF, Brodie MJ, et al. Revised terminology and concepts for organization of seizures and epilepsies: report of the ILAE Commission on Classification and Terminology, 2005-2009. Epilepsia 2010;51(4):676-685

14 Farwell KD, Shahmirzadi L, El-Khechen D, et al. Enhanced utility of family-centered diagnostic exome sequencing with inheritance model-based analysis: results from 500 unselected families with undiagnosed genetic conditions. Genet Med 2014; e-pub ahead of print

15 Taylor RW, Pyle A, Griffin H, et al. Use of whole-exome sequencing to determine the genetic basis of multiple mitochondrial respiratory chain complex deficiencies. JAMA 2014;312(1):68-77

16 Schaefer AM, McFarland R, Blakely EL, et al. Prevalence of mitochondrial DNA disease in adults. Ann Neurol 2008;63(1):35-39

17 Han JY, Sung JJ, Park HK, Yoon BN, Lee KW. Adult onset Leigh syndrome with mitochondrial DNA $8344 \mathrm{~A}>\mathrm{G}$ mutation. J Clin Neurosci 2014;21(11):2009-2011

18 McKelvie P, Infeld B, Marotta R, Chin J, Thorburn D, Collins S. Late-adult onset Leigh syndrome. J Clin Neurosci 2012;19(2): 195-202

19 Rahman S, Blok RB, Dahl HH, et al. Leigh syndrome: clinical features and biochemical and DNA abnormalities. Ann Neurol 1996;39(3):343-351

20 Lee HF, Tsai CR, Chi CS, Lee HJ, Chen CC. Leigh syndrome: clinical and neuroimaging follow-up. Pediatr Neurol 2009;40(2):88-93
21 Naess K, Freyer C, Bruhn H, et al. MtDNA mutations are a common cause of severe disease phenotypes in children with Leigh syndrome. Biochim Biophys Acta 2009;1787(5):484-490

22 Arii J, Tanabe Y. Leigh syndrome: serial MR imaging and clinical follow-up. AJNR Am J Neuroradiol 2000;21(8):1502-1509

23 Ruhoy IS, Saneto RP. The genetics of Leigh syndrome and its implications for clinical practice and risk management. Appl Clin Genet 2014;7:221-234

24 Saneto RP, Cohen BH, Copeland WC, Naviaux RK. Alpers-Huttenlocher syndrome. Pediatr Neurol 2013;48(3):167-178

25 Hunter MF, Peters H, Salemi R, Thorburn D, Mackay MT. Alpers syndrome with mutations in POLG: clinical and investigative features. Pediatr Neurol 2011;45(5):311-318

26 McCoy B, Owens C, Howley R, et al. Partial status epilepticus - rapid genetic diagnosis of Alpers' disease. Eur J Paediatr Neurol 2011; 15(6):558-562

27 Wolf NI, Rahman S, Schmitt B, et al. Status epilepticus in children with Alpers' disease caused by POLG1 mutations: EEG and MRI features. Epilepsia 2009;50(6):1596-1607

28 Saneto RP, Lee IC, Koenig MK, et al. POLG DNA testing as an emerging standard of care before instituting valproic acid therapy for pediatric seizure disorders. Seizure 2010;19(3):140-146

29 Lupashko S, Malik S, Donahue D, Hernandez A, Perry MS. Palliative functional hemispherectomy for treatment of refractory status epilepticus associated with Alpers' disease. Childs Nerv Syst 2011; 27(8):1321-1323

30 Tanji K, Kaufmann P, Naini AB, et al. A novel tRNA(Val) mitochondrial DNA mutation causing MELAS. J Neurol Sci 2008;270(1-2): 23-27

31 Tzoulis C, Bindoff LA. Melas associated with mutations in the polg1 gene. Neurology 2008;70(13):1054, author reply 1054-1055

32 Deschauer M, Tennant S, Rokicka A, et al. MELAS associated with mutations in the POLG1 gene. Neurology 2007;68(20):1741-1742

33 Karkare S, Merchant S, Solomon G, Engel M, Kosofsky B. MELAS with A3243G mutation presenting with occipital status epilepticus. J Child Neurol 2009;24(12):1564-1567

34 Silvestri G, Moraes CT, Shanske S, Oh SJ, DiMauro S. A new mtDNA mutation in the tRNA(Lys) gene associated with myoclonic epilepsy and ragged-red fibers (MERRF). Am J Hum Genet 1992; 51(6):1213-1217

35 Van Goethem G, Mercelis R, Löfgren A, et al. Patient homozygous for a recessive POLG mutation presents with features of MERRF. Neurology 2003;61(12):1811-1813

36 Romaniuk L, Turnbull DM, Whittaker RG. Hyperventilation during the EEG is safe in mitochondrial disease. Clin Neurophysiol 2011; 122(6):1270-1271

37 OMIM. 174763: Polymerase, DNA, Gamma; POLG. 25/10/2013 ed. Online Mendelian Inheritance in Man;2013

38 Tang S, Wang J, Lee NC, et al. Mitochondrial DNA polymerase gamma mutations: an ever expanding molecular and clinical spectrum. J Med Genet 2011;48(10):669-681

39 Tzoulis C, Engelsen BA, Telstad W, et al. The spectrum of clinical disease caused by the A467T and W748S POLG mutations: a study of 26 cases. Brain 2006;129(Pt 7):1685-1692

40 Horvath R, Hudson G, Ferrari G, et al. Phenotypic spectrum associated with mutations of the mitochondrial polymerase gamma gene. Brain 2006;129(Pt 7):1674-1684

41 Hakonen AH, Davidzon G, Salemi R, et al. Abundance of the POLG disease mutations in Europe, Australia, New Zealand, and the United States explained by single ancient European founders. Eur J Hum Genet 2007;15(7):779-783

42 Engelsen BA, Tzoulis C, Karlsen B, et al. POLG1 mutations cause a syndromic epilepsy with occipital lobe predilection. Brain 2008; 131(Pt 3):818-828

43 lizuka T, Sakai F, Kan S, Suzuki N. Slowly progressive spread of the stroke-like lesions in MELAS. Neurology 2003;61(9):1238-1244 
44 Lönnqvist T, Paetau A, Valanne L, Pihko H. Recessive twinkle mutations cause severe epileptic encephalopathy. Brain 2009; 132(Pt 6):1553-1562

45 Kwan P, Sander JW. The natural history of epilepsy: an epidemiological view. J Neurol Neurosurg Psychiatry 2004;75(10): 1376-1381

46 Keränen T, Kuusisto H. NARP syndrome and adult-onset generalised seizures. Epileptic Disord 2006;8(3):200-203

47 Kosinski C, Mull M, Lethen H, Töpper R. Evidence for cardioembolic stroke in a case of Kearns-Sayre syndrome. Stroke 1995;26(10): 1950-1952

48 Furuya H, Sugimura T, Yamada T, Hayashi K, Kobayashi T. [A case of incomplete Kearns-Sayre syndrome with a stroke like episode]. Rinsho Shinkeigaku 1997;37(8):680-684

49 Niehusmann P, Surges R, von Wrede RD, et al. Mitochondrial dysfunction due to Leber's hereditary optic neuropathy as a cause of visual loss during assessment for epilepsy surgery. Epilepsy Behav 2011;20(1):38-43

50 Grazina MM, Diogo LM, Garcia PC, et al. Atypical presentation of Leber's hereditary optic neuropathy associated to mtDNA $11778 \mathrm{G}>\mathrm{A}$ point mutation-A case report. Eur J Paediatr Neurol 2007;11(2):115-118

51 Mazzaccara C, Iafusco D, Liguori R, et al. Mitochondrial diabetes in children: seek and you will find it. PLoS ONE 2012;7(4):e34956

52 Delmiro A, Rivera H, García-Silva MT, et al. Whole-exome sequencing identifies a variant of the mitochondrial MT-ND1 gene associated with epileptic encephalopathy: west syndrome evolving to Lennox-Gastaut syndrome. Hum Mutat 2013;34(12):1623-1627

53 Elia M, Musumeci SA, Ferri R, et al. Leigh syndrome and partial deficit of cytochrome c oxidase associated with epilepsia partialis continua. Brain Dev 1996;18(3):207-211

54 Yang PF, Jia YZ, Lin Q et al. Intractable occipital lobe epilepsy: clinical characteristics, surgical treatment, and a systematic review of the literature. Acta Neurochir (Wien) 2015;157(1):63-75

55 Adcock JE, Panayiotopoulos CP. Occipital lobe seizures and epilepsies. J Clin Neurophysiol 2012;29(5):397-407

56 Hallmann K, Zsurka G, Moskau-Hartmann S, et al. A homozygous splice-site mutation in CARS2 is associated with progressive myoclonic epilepsy. Neurology 2014;83(23):2183-2187

57 Girard JM, Turnbull J, Ramachandran N, Minassian BA. Progressive myoclonus epilepsy. Handb Clin Neurol 2013;113:1731-1736

58 Castro-Gago M, Blanco-Barca MO, Gómez-Lado C, Eirís-Puñal J, Campos-González Y, Arenas-Barbero J. Respiratory chain complex I deficiency in an infant with Ohtahara syndrome. Brain Dev 2009; 31(4):322-325

59 Tsuji M, Kuroki S, Maeda H, et al. Leigh syndrome associated with West syndrome. Brain Dev 2003;25(4):245-250

60 Kang HC, Kim HD, Lee YM, Han SH. Landau-Kleffner syndrome with mitochondrial respiratory chain-complex I deficiency. Pediatr Neurol 2006;35(2):158-161

61 Fujimoto S, Mizuno K, Shibata H, et al. Serial electroencephalographic findings in patients with MELAS. Pediatr Neurol 1999; 20(1):43-48

62 Nesbitt V, Alston CL, Blakely EL, et al. A national perspective on prenatal testing for mitochondrial disease. Eur J Hum Genet 2014; 22(11):1255-1259

63 Jackson MJ, Schaefer JA, Johnson MA, Morris AA, Turnbull DM, Bindoff LA. Presentation and clinical investigation of mitochondrial respiratory chain disease. A study of 51 patients. Brain 1995; 118(Pt 2):339-357
64 Davis RL, Liang C, Edema-Hildebrand F, Riley C, Needham M, Sue CM. Fibroblast growth factor 21 is a sensitive biomarker of mitochondrial disease. Neurology 2013;81(21):1819-1826

65 Suomalainen A, Elo JM, Pietiläinen KH, et al. FGF-21 as a biomarker for muscle-manifesting mitochondrial respiratory chain deficiencies: a diagnostic study. Lancet Neurol 2011;10(9):806-818

66 Research WTCfM. Stroke-like episodes in adult mitochondrial disease: investigation and management. Available at: http://www.newcastle-mitochondria.com/wp-content/uploads/2012/09/Stroke-LikeEpisodes-Guidelines.pdf2013. Accessed January 27, 2015

67 Pfeffer G, Majamaa K, Turnbull DM, Thorburn D, Chinnery PF. Treatment for mitochondrial disorders. Cochrane Database Syst Rev 2012;4:CD004426

68 Finsterer J, Zarrouk Mahjoub S. Mitochondrial toxicity of antiepileptic drugs and their tolerability in mitochondrial disorders. Expert Opin Drug Metab Toxicol 2012;8(1):71-79

69 Shorvon S, Ferlisi M. The treatment of super-refractory status epilepticus: a critical review of available therapies and a clinical treatment protocol. Brain 2011;134(Pt 10):2802-2818

70 Visser NA, Braun KP, Leijten FS, van Nieuwenhuizen O, Wokke JH, van den Bergh WM. Magnesium treatment for patients with refractory status epilepticus due to POLG1-mutations. J Neurol 2011;258(2):218-222

71 Howard RS, Russell S, Losseff N, et al. Management of mitochondrial disease on an intensive care unit. OJM 1995;88(3):197-207

72 Ferlisi M, Shorvon S. The outcome of therapies in refractory and super-refractory convulsive status epilepticus and recommendations for therapy. Brain 2012;135(Pt 8):2314-2328

73 Martikainen MH, Päivärinta M, Jääskeläinen S, Majamaa K. Successful treatment of POLG-related mitochondrial epilepsy with antiepileptic drugs and low glycaemic index diet. Epileptic Disord 2012;14(4):438-441

74 Kang HC, Lee YM, Kim HD, Lee JS, Slama A. Safe and effective use of the ketogenic diet in children with epilepsy and mitochondrial respiratory chain complex defects. Epilepsia 2007;48(1):82-88

75 Joshi CN, Greenberg CR, Mhanni AA, Salman MS. Ketogenic diet in Alpers-Huttenlocher syndrome. Pediatr Neurol 2009;40(4):314-316

76 Matthews Friends: ketogenic dietary therapies. Available at: www.matthewsfriends.org/. Accessed November 20, 2014

77 Arthur TM, Saneto RP, de Menezes MS, et al. Vagus nerve stimulation in children with mitochondrial electron transport chain deficiencies. Mitochondrion 2007;7(4):279-283

78 Carter BS. Comment to the paper: Palliative functional hemispherectomy for treatment of refractory status epilepticus associated with Alper's disease. Childs Nerv Syst 2011;27(8):1325-1326

79 Duchowny M. Comment to the paper: palliative functional hemispherectomy for treatment of refractory status epilepticus associated with Alpers' disease. Childs Nerv Syst 2011;27(8):1327-1328

80 Billings JA. The end-of-life family meeting in intensive care part I: indications, outcomes, and family needs. J Palliat Med 2011;14(9): 1042-1050

81 Billings JA, Block SD. The end-of-life family meeting in intensive care part III: a guide for structured discussions. J Palliat Med 2011; 14(9):1058-1064

82 Richardson J, Irving L, Hyslop LA, et al. Concise reviews: assisted reproductive technologies to prevent transmission of mitochondrial DNA disease. Stem Cells 2015;33(3):639-645

83 Pfeffer G, Horvath R, Klopstock T, et al. New treatments for mitochondrial disease-no time to drop our standards. Nat Rev Neurol 2013;9(8):474-481 